\title{
Infrared study of IRAS 18316-0602 star-forming region
}

\author{
Andreasyan D., Azatyan N, Nikoghosyan E., Harutyunian H., and Baghdasaryan D. \\ Byurakan Astrophysical Observatory, Aragatsotn region, Byurakan vil., Armenia
}

\begin{abstract}
We present the results of the investigation of ISM and the young stellar population in the IRAS 18316-0602 star-forming region which is referred to as UC HII (G25.65+1.05). Single temperature modified blackbody model shows that values of $\mathrm{N}\left(\mathrm{H}_{2}\right)$ hydrogen column density and $\mathrm{T}_{d}$ dust temperature are in ranges $2-7 \times 10^{23} \mathrm{~cm}^{-2}$ and $12-30 \mathrm{~K}$, respectively. The analysis of infrared photometric data allowed to reveal about 50 young stellar objects in the G25.65+1.05 UC HII region.
\end{abstract}

Keywords: stars: pre-main sequence - infrared: stars - radiative transfer - ISM: hydrogen column density, dust temperature

\section{Introduction}

IRAS 18316-0602 (also known as G25.65+1.05 UC HII region) is associated with an irregular compact radio source, first identified at $3.6 \mathrm{~cm}$ by Kurtz et al. (1994). The radio peak is coincident with an unresolved infrared (IR) source, identified as a young B1V star with large $\mathrm{K}$ band excess (Zavagno et al., 2002). Based on the CO observations, Shepherd \& Churchwell (1996) detected an energetic bipolar outflow centred on the radio source. It is also closely associated with $\mathrm{NH}_{3}$ emission (Molinari et al., 1996) and strong $\mathrm{CH}_{3} \mathrm{OH}$ (Szymczak et al., 2000) and $\mathrm{H}_{2} \mathrm{O}$ maser (Kurtz \& Hofner, 2005) emissions. The distance to the source is an open question. Molecular line observations in most cases argue in favor of the near kinematic distance $\sim 3.17 \mathrm{kpc}$ (Molinari et al., 1996). In contrast, HI selfabsorption toward the source suggests a far kinematic distance of $12.5 \mathrm{kpc}$ (Green \& McClure-Griffiths, 2011).

IR study of the star-forming region in G25.65+1.05 was carried out in two main directions: determination of ISM physical parameters (hydrogen column density $\left(\mathrm{N}\left(\mathrm{H}_{2}\right)\right)$ and dust temperature $\left(\mathrm{T}_{d}\right)$, as well as the identification of the young stellar population in this region.

\section{Method}

To obtain the physical parameters like $\mathrm{N}\left(\mathrm{H}_{2}\right)$ and $\mathrm{T}_{d}$, the thermal emission from cold dust lying in the Herschel FIR optically thin bands $(160-500 \mu \mathrm{m})$ can be used (Battersby et al., 2011, Hildebrand, 1983). For this task, Level 2.5 processed Herschel images were used. The initial processing of the images was carried out with HIPE software. To obtain the $\mathrm{N}\left(\mathrm{H}_{2}\right)$ and $\mathrm{T}_{d}$, we used the modified single-temperature blackbody fitting which was subsequently carried out on a pixel-by-pixel basis using the following formula:

$$
S_{\nu}=B_{\nu}\left(\nu, T_{d}\right) \Omega\left(1-e^{-\tau(\nu)}\right)
$$

with

$$
\tau(\nu)=\mu_{H_{2}} m_{H} k_{\nu} N\left(H_{2}\right),
$$

where $\nu$ is the frequency, $S_{\nu}(\nu)$ is the observed flux density, $B_{\nu}\left(\nu, T_{d}\right)$ is the Planck function, $T_{d}$ is the dust temperature, $\Omega$ is the solid angle in steradians from where the flux is obtained, $\tau(\nu)$ is the optical

*derenik@bao.sci.am, Corresponding author 

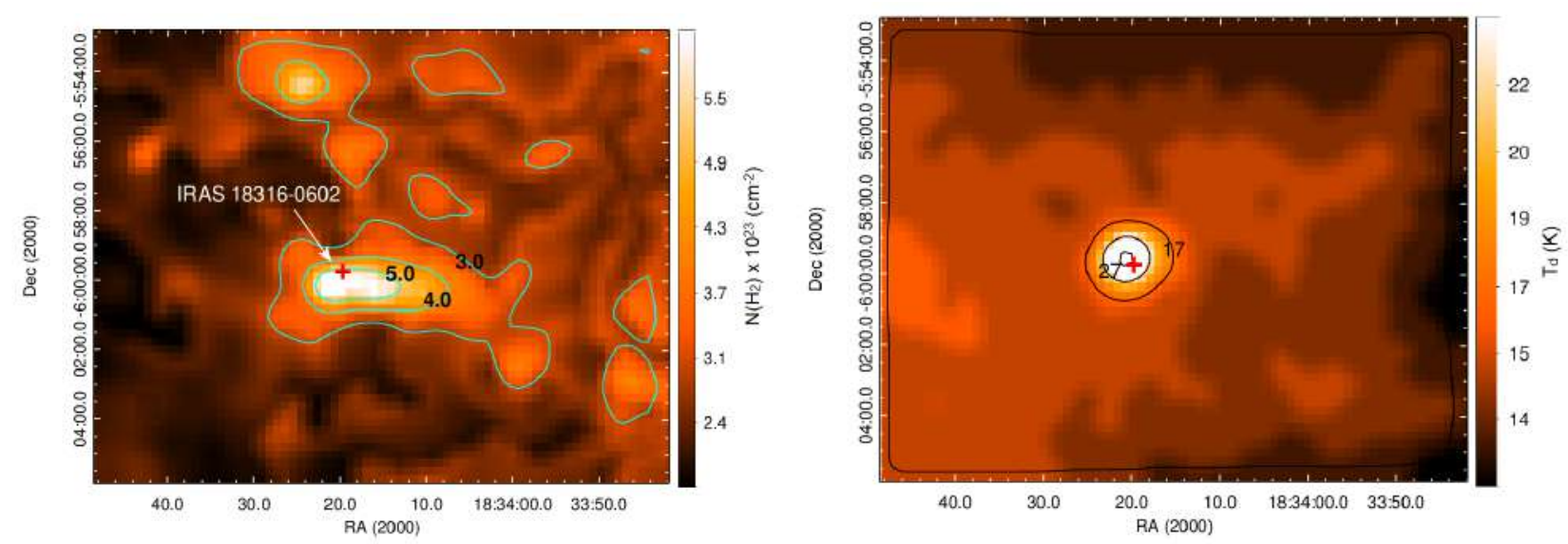

Figure 1. Maps of $\mathrm{N}\left(\mathrm{H}_{2}\right)$ (left panel) and $\mathrm{T}_{d}$ (right panel) of the region surrounding G25.65+1.05 UCHII object. On the $\mathrm{N}\left(\mathrm{H}_{2}\right)$ map, the isodenses corresponding to the values of $3.0 \times 10^{23} \mathrm{~cm}^{-2}$, $4.0 \times 10^{23} \mathrm{~cm}^{-2}$, and $5.0 \times 10^{23} \mathrm{~cm}^{-2}$ are shown. On the $\mathrm{T}_{d}$ map, isotherms corresponding to the values of $17 \mathrm{~K}$ and $27 \mathrm{~K}$ are shown. The positions of IRAS 18316-0602 are marked by crosses.

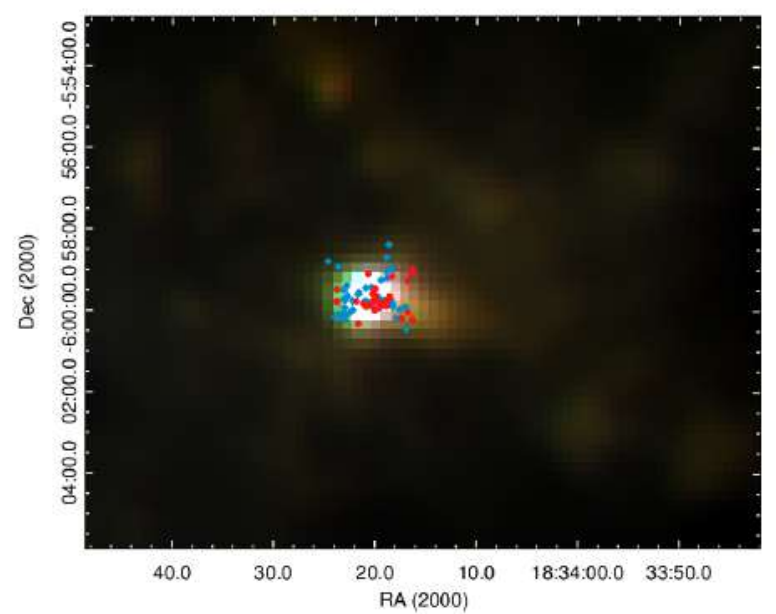

Figure 2. Distribution of YSOs in the region on Herschel $500 \mu \mathrm{m}$ image. Class I and Class II objects are indicated by filled red and blue circles, respectively.

depth, $\mu_{H_{2}}$ is the mean molecular weight (adopted as 2.8 here), $m_{H}$ is the mass of hydrogen, $k_{\nu}$ is the dust opacity, and $\mathrm{N}\left(\mathrm{H}_{2}\right)$ is the hydrogen column density. For opacity, we adopted a functional form of $k_{\nu}=0.1(\nu / 1000 \mathrm{GHz})^{\beta} \mathrm{cm}^{2} \mathrm{~g}^{-1}$, with $\beta=2$ (Hildebrand, 1983). For each pixel, equation (1) was fitted using the four data points $(160,250,350$, and $500 \mu \mathrm{m})$ keeping $\mathrm{T}_{d}$ and $\mathrm{N}\left(\mathrm{H}_{2}\right)$ as free parameters.

For the search, identification, and classification of the young stellar population of the UC HII region, we used their NIR (UKIDSS), MIR (Spitzer, WISE), and FIR (Herschel) photometric data. When selecting potential members of the cluster from stars located in the direction of the molecular cloud, we proceeded from the assumption that the overwhelming majority of the members of the considered star-forming region are young stellar objects (YSO). According to the star formation theory, the IR excess of YSOs is caused by a circumstellar disk and gas-dust envelope, which are known as the main characteristics of YSOs (Hartmann, 2009, Lada \& Lada, 2003). Therefore, according to the IR excess, it is possible to carry out the selection of Class I and Class II evolutionary stage YSOs. One of the most powerful tools for identifying YSO candidates via IR excess is their location on color-color (c-c) diagrams. The choice of colors depends on the available data. 


\section{Results and Conclusion}

The $\mathrm{N}\left(\mathrm{H}_{2}\right)$ and $\mathrm{T}_{d}$ of the wider region surrounding $\mathrm{G} 25.65+1.05 \mathrm{UC}$ HII region are shown in Figure 1. We can see that the column density distribution has three, well distinguished concentrations. In general, within UC HII region the column density varies from $\sim 3.0 \times 10^{23}$ to $7.0 \times 10^{23} \mathrm{~cm}^{-2}$, which corresponds to the values of $\mathrm{N}\left(\mathrm{H}_{2}\right)$ in other UC HII regions (Churchwell et al., 2010). The $\mathrm{T}_{d}$ distribution has spherical symmetry. It decreases from the center to the periphery, from $30 \mathrm{~K}$ to $15 \mathrm{~K}$. The maxima of both density and temperature coincide with the position of the IRAS source.

The search, identification, and classification of the young stellar population of the molecular cloud using their NIR, MIR, and FIR photometric data was based on one of the main properties of young stars, namely the IR excess due to the presence of circumstellar disks and envelopes. In total, relative to the stellar objects' position in the c-c diagrams, we managed to identify 69 YSOs with different evolutionary stages (Class I and II). The YSOs are located directly in the vicinity of IRAS 18316-0602, forming a young stellar cluster.

The results obtained undoubtedly create a prerequisite for further detailed studies of this starforming region.

\section{Acknowledgements}

The authors are grateful to the organizers for holding the International Symposium "Astronomical Surveys and Big Data 2" and for publishing the paper.

\section{References}

Battersby C., et al., 2011, , 535, A128

Churchwell E., Sievers A., Thum C., 2010, A\&A, 513, A9

Green J. A., McClure-Griffiths N. M., 2011, MNRAS, 417, 2500

Hartmann L., 2009, Astrophysics and Space Science Proceedings, 13, 23

Hildebrand R. H., 1983, QJRAS, 24, 267

Kurtz S., Hofner P., 2005, AJ, 130, 711

Kurtz S., Churchwell E., Wood D. O. S., 1994, ApJS, 91, 659

Lada C. J., Lada E. A., 2003, ARA\&A, 41, 57

Molinari S., Brand J., Cesaroni R., Palla F., 1996, A\&A, 308, 573

Shepherd D. S., Churchwell E., 1996, ApJ, 472, 225

Szymczak M., Hrynek G., Kus A. J., 2000, A\&AS, 143, 269

Zavagno A., Deharveng L., Nadeau D., Caplan J., 2002, A\&A, 394, 225 